\title{
Nonlinear Optical Properties of XPh4 (X = B-, C, N+, P+): A New Class of Molecules with a Negative Third-Order Polarizability
}

Rebecca L. Gieseking, Trenton R. Ensley, Honghua Hu, David J. Hagan, Chad Risko, Eric W. Van Stryland, and Jean-Luc Bredas

J. Am. Chem. Soc., Just Accepted Manuscript • DOI: 10.1021/jacs.5b04377 • Publication Date (Web): 22 Jun 2015

Downloaded from http://pubs.acs.org on June 28, 2015

\section{Just Accepted}

"Just Accepted" manuscripts have been peer-reviewed and accepted for publication. They are posted online prior to technical editing, formatting for publication and author proofing. The American Chemical Society provides "Just Accepted" as a free service to the research community to expedite the dissemination of scientific material as soon as possible after acceptance. "Just Accepted" manuscripts appear in full in PDF format accompanied by an HTML abstract. "Just Accepted" manuscripts have been fully peer reviewed, but should not be considered the official version of record. They are accessible to all readers and citable by the Digital Object Identifier (DOI®). "Just Accepted" is an optional service offered to authors. Therefore, the "Just Accepted" Web site may not include all articles that will be published in the journal. After a manuscript is technically edited and formatted, it will be removed from the "Just Accepted" Web site and published as an ASAP article. Note that technical editing may introduce minor changes to the manuscript text and/or graphics which could affect content, and all legal disclaimers and ethical guidelines that apply to the journal pertain. ACS cannot be held responsible for errors or consequences arising from the use of information contained in these "Just Accepted" manuscripts. 


\title{
Nonlinear Optical Properties of $\mathrm{XPh}_{4}\left(\mathrm{X}=\mathrm{B}^{-}, \mathrm{C}, \mathbf{N}^{+}, \mathbf{P}^{+}\right)$:
}

\section{A New Class of Molecules with a Negative Third-Order Polarizability}

\author{
Rebecca L. Gieseking ${ }^{1}$, Trenton R. Ensley ${ }^{2}$, Honghua Hu ${ }^{2}$, David J. Hagan ${ }^{2}$, Chad Risko ${ }^{1,3}$, \\ Eric W. Van Stryland ${ }^{2, *}$, and Jean-Luc Brédas ${ }^{1,4, *}$
}

${ }^{I}$ School of Chemistry and Biochemistry and Center for Organic Photonics and Electronics Georgia Institute of Technology Atlanta, Georgia 30332-0400, USA

${ }^{2}$ CREOL, The College of Optics and Photonics University of Central Florida Orlando, Florida 32816, USA

${ }^{3}$ Department of Chemistry and Center for Applied Energy Research University of Kentucky Lexington, Kentucky 40506-0055, USA

${ }^{4}$ Division of Physical Sciences and Engineering and Solar \& Photovoltaics Engineering Research Center King Abdullah University of Science and Technology Thuwal 23955-6900, Kingdom of Saudi Arabia

\footnotetext{
*Corresponding Authors:

ewvs@creol.ucf.edu, jean-luc.bredas@kaust.edu.sa
} 
Abstract

Organic $\pi$-conjugated materials have been widely used for a variety of nonlinear optical (NLO) applications. Molecules with negative real components $\operatorname{Re}(\gamma)$ of the third-order polarizability, which leads to nonlinear refraction in macroscopic systems, have important benefits for several NLO applications. However, few organic systems studied to date have negative $\operatorname{Re}(\gamma)$ in the long wavelength limit, and all inorganic materials show positive nonlinear refraction in this limit. Here, we introduce a new class of molecules of the form $\mathrm{X}\left(\mathrm{C}_{6} \mathrm{H}_{5}\right)_{4}$, where $\mathrm{X}=\mathrm{B}^{-}, \mathrm{C}, \mathrm{N}^{+}$, and $\mathrm{P}^{+}$, that have negative $\operatorname{Re}(\gamma)$. The molecular mechanism for the NLO properties in these systems is very different from those in typical linear conjugated systems: these systems have a band of excited states involving single-electron excitations within the $\pi$-system, several of which have significant coupling to the ground state. Thus, $\operatorname{Re}(\gamma)$ cannot be understood in terms of a simplified essential-state model and must be analyzed in the context of the full sum-over-states expression. Although $\operatorname{Re}(\gamma)$ is significantly smaller than that of other commonly-studied NLO chromophores, the introduction of a new molecular architecture offering the potential for a negative $\operatorname{Re}(\gamma)$ introduces new avenues of molecular design for NLO applications. 


\section{Introduction}

Materials based on organic $\pi$-conjugated chromophores have been successfully applied in many nonlinear optical (NLO) applications. For instance, molecules with large second-order polarizabilities have been used in electro-optic ${ }^{1-4}$ and second-harmonic imaging applications, ${ }^{5,6}$ while materials with large imaginary parts of the third-order polarizability have been used in two-photon imaging ${ }^{7-9}$ and optical power limiting. ${ }^{10,11}$ However, the development of organic materials for applications such as (i) all-optical switching (AOS), which requires very large real parts of the molecular third-order polarizability $|\operatorname{Re}(\gamma)|$ and small imaginary parts $\operatorname{Im}(\gamma)$; or (ii) data transmission, which requires extremely small $\operatorname{Re}(\gamma)$ and $\operatorname{Im}(\gamma)$, has been limited due to challenges in achieving both the necessary molecular and materials properties. $\operatorname{Re}(\gamma)$ is directly proportional to the nonlinear refractive index $\left(\mathrm{n}_{2}\right)$ of the material, whereas $\operatorname{Im}(\gamma)$ is directly proportional to the two-photon absorption (2PA) coefficient.

The development of molecules and materials with negative $\operatorname{Re}(\gamma)$ is critical for both AOS and datatransmission applications. For AOS applications, few molecular materials have the required figure-ofmerit $(|\operatorname{Re}(\gamma) / \operatorname{Im}(\gamma)|>4 \pi) .{ }^{12-14}$ Although AOS devices can in principle be made using materials with either a positive or negative value of $\operatorname{Re}(\gamma)$, in practice a negative $\operatorname{Re}(\gamma)$ can provide important benefits. Since materials with positive $\operatorname{Re}(\gamma)$ are self-focusing, ${ }^{15-17}$ the peak intensity of each light signal increases as the pulse travels through the material. This limits the laser intensity that can be used without risking dielectric breakdown of the NLO material. ${ }^{18}$ As the change in refractive index is linearly dependent on the intensity of light, reducing light intensity would require a larger $\operatorname{Re}(\gamma)$ or a longer interaction length for a functional device. Materials with negative $\operatorname{Re}(\gamma)$ are self-defocusing, which may alleviate these challenges.

While large nonlinearities in fibers are required for applications such as AOS, such effects are detrimental for data transmission and for fiber lasers. ${ }^{19,20}$ As an example, one can consider liquid core optical fibers (LCOFs). Since the solutions typically used in the cores have positive $\mathrm{n}_{2}$, the propagation of high intensity 
pulses in these fibers can induce self-phase modulation (SPM) $)^{21-23}$ that can spectrally broaden the pulses and eventually lead to supercontinuum generation. ${ }^{24,25}$ By doping the core with a small amount of a negative $\operatorname{Re}(\gamma)$ material, the deleterious effects of SPM can be drastically reduced with negligible effects on the linear refractive index.

Although molecules and materials possessing negative $\operatorname{Re}(\gamma)$, and hence negative $n_{2}$, could provide substantial benefits for several device applications, to date relatively few classes of materials have been shown to have negative $\operatorname{Re}(\gamma) \cdot{ }^{26-34}$ Without exception, inorganic materials show positive $\mathrm{n}_{2}$ in the long wavelength limit, ${ }^{35,36}$ leaving organic materials as the sole route to materials with negative $\mathrm{n}_{2}$. Developing new classes of molecules with negative $\operatorname{Re}(\gamma)$ requires understanding the molecular processes that affect the sign and magnitude of $\gamma$. The molecular polarizabilities can be described in terms of the response of the molecules to the electric field of light. When an electric field $\vec{F}$ is applied, the molecular dipole moment $\vec{\mu}$ can be expressed in a power series expansion as:

$$
\vec{\mu}(\vec{F})=\vec{\mu}_{0}+\alpha \cdot \vec{F}+\beta \cdot \vec{F} \vec{F}+\gamma \cdot \vec{F} \vec{F} \vec{F}+\cdots
$$

1

where $\vec{\mu}_{0}$ represents the permanent molecular dipole moment in the absence of an applied electric field; $\alpha$, the linear polarizability; and $\beta$, the second-order polarizability. If the electric field of light is treated as a perturbation, $\gamma$ can be expressed in terms of the molecular properties as a sum-over-(electronic) states (SOS) expression: ${ }^{37}$ 
$=I_{\mathrm{p}, \mathrm{q}, \mathrm{r}}$

$\times\left\{\sum_{u \neq g} \sum_{v \neq g} \sum_{w \neq g}\left[\frac{\left\langle\psi_{\mathrm{g}}\left|\hat{\mu}^{\mathrm{i}}\right| \psi_{\mathrm{u}}\right\rangle\left\langle\psi_{\mathrm{u}}\left|\bar{\mu}^{\mathrm{l}}\right| \psi_{\mathrm{v}}\right\rangle\left\langle\psi_{\mathrm{v}}\left|\bar{\mu}^{\mathrm{k}}\right| \psi_{\mathrm{w}}\right\rangle\left\langle\psi_{\mathrm{w}}\left|\hat{\mu}^{\mathrm{j}}\right| \psi_{\mathrm{g}}\right\rangle}{\left(E_{\mathrm{gu}}-i \Gamma_{\mathrm{gu}}-\hbar \omega\right)\left(E_{\mathrm{gv}}-i \Gamma_{\mathrm{gv}}-\hbar \omega_{\mathrm{p}}-\hbar \omega_{\mathrm{q}}\right)\left(E_{\mathrm{gw}}-i \Gamma_{\mathrm{gw}}-\hbar \omega_{\mathrm{p}}\right)}\right.\right.$

$+\frac{\left\langle\psi_{\mathrm{g}}\left|\hat{\mu}^{1}\right| \psi_{\mathrm{u}}\right\rangle\left\langle\psi_{\mathrm{u}}\left|\bar{\mu}^{\mathrm{i}}\right| \psi_{\mathrm{v}}\right\rangle\left\langle\psi_{\mathrm{v}}\left|\bar{\mu}^{\mathrm{k}}\right| \psi_{\mathrm{w}}\right\rangle\left\langle\psi_{\mathrm{w}}\left|\hat{\mu}^{\mathrm{j}}\right| \psi_{\mathrm{g}}\right\rangle}{\left(E_{\mathrm{gu}}-i \Gamma_{\mathrm{gu}}+\hbar \omega_{\mathrm{r}}\right)\left(E_{\mathrm{gv}}-i \Gamma_{\mathrm{gv}}-\hbar \omega_{\mathrm{p}}-\hbar \omega_{\mathrm{q}}\right)\left(E_{\mathrm{gw}}-i \Gamma_{\mathrm{gw}}-\hbar \omega_{\mathrm{p}}\right)}$

$+\frac{\left\langle\psi_{\mathrm{g}}\left|\hat{\mu}^{\mathrm{j}}\right| \psi_{\mathrm{u}}\right\rangle\left\langle\psi_{\mathrm{u}}\left|\bar{\mu}^{\mathrm{k}}\right| \psi_{\mathrm{v}}\right\rangle\left\langle\psi_{\mathrm{v}}\left|\bar{\mu}^{\mathrm{i}}\right| \psi_{\mathrm{w}}\right\rangle\left\langle\psi_{\mathrm{w}}\left|\hat{\mu}^{1}\right| \psi_{\mathrm{g}}\right\rangle}{\left(E_{\mathrm{gu}}-i \Gamma_{\mathrm{gu}}+\hbar \omega_{\mathrm{p}}\right)\left(E_{\mathrm{gv}}-i \Gamma_{\mathrm{gv}}+\hbar \omega_{\mathrm{p}}+\hbar \omega_{\mathrm{q}}\right)\left(E_{\mathrm{gw}}-i \Gamma_{\mathrm{gw}}-\hbar \omega_{\mathrm{r}}\right)}$

$\left.+\frac{\left\langle\psi_{\mathrm{g}}\left|\hat{\mu}^{\mathrm{j}}\right| \psi_{\mathrm{u}}\right\rangle\left\langle\psi_{\mathrm{u}}\left|\bar{\mu}^{\mathrm{k}}\right| \psi_{\mathrm{v}}\right\rangle\left\langle\psi_{\mathrm{v}}\left|\bar{\mu}^{\mathrm{l}}\right| \psi_{\mathrm{w}}\right\rangle\left\langle\psi_{\mathrm{w}}\left|\hat{\mu}^{\mathrm{i}}\right| \psi_{\mathrm{g}}\right\rangle}{\left(E_{\mathrm{gu}}-i \Gamma_{\mathrm{gu}}+\hbar \omega_{\mathrm{p}}\right)\left(E_{\mathrm{gv}}-i \Gamma_{\mathrm{gv}}+\hbar \omega_{\mathrm{p}}+\hbar \omega_{\mathrm{q}}\right)\left(E_{\mathrm{gw}}-i \Gamma_{\mathrm{gw}}+\hbar \omega\right)}\right]$

$-\sum_{u \neq g} \sum_{w \neq g}\left[\frac{\left\langle\psi_{\mathrm{g}}\left|\hat{\mu}^{\mathrm{i}}\right| \psi_{\mathrm{u}}\right\rangle\left\langle\psi_{\mathrm{u}}\left|\hat{\mu}^{1}\right| \psi_{\mathrm{g}}\right\rangle\left\langle\psi_{\mathrm{g}}\left|\hat{\mu}^{\mathrm{k}}\right| \psi_{\mathrm{w}}\right\rangle\left\langle\psi_{\mathrm{w}}\left|\hat{\mu}^{\mathrm{j}}\right| \psi_{\mathrm{g}}\right\rangle}{\left(E_{\mathrm{gu}}-i \Gamma_{\mathrm{gu}}-\hbar \omega\right)\left(E_{\mathrm{gu}}-i \Gamma_{\mathrm{gu}}-\hbar \omega_{\mathrm{r}}\right)\left(E_{\mathrm{gw}}-i \Gamma_{\mathrm{gw}}-\hbar \omega_{\mathrm{p}}\right)}\right.$

$+\frac{\left\langle\psi_{\mathrm{g}}\left|\hat{\mu}^{\mathrm{i}}\right| \psi_{\mathrm{u}}\right\rangle\left\langle\psi_{\mathrm{u}}\left|\hat{\mu}^{1}\right| \psi_{\mathrm{g}}\right\rangle\left\langle\psi_{\mathrm{g}}\left|\hat{\mu}^{\mathrm{k}}\right| \psi_{\mathrm{w}}\right\rangle\left\langle\psi_{\mathrm{w}}\left|\hat{\mu}^{\mathrm{j}}\right| \psi_{\mathrm{g}}\right\rangle}{\left(E_{\mathrm{gu}}-i \Gamma_{\mathrm{gu}}-\hbar \omega_{\mathrm{r}}\right)\left(E_{\mathrm{gw}}-i \Gamma_{\mathrm{gw}}+\hbar \omega_{\mathrm{q}}\right)\left(E_{\mathrm{gw}}-i \Gamma_{\mathrm{gw}}-\hbar \omega_{\mathrm{p}}\right)}$

$+\frac{\left\langle\psi_{\mathrm{g}}\left|\hat{\mu}^{1}\right| \psi_{\mathrm{u}}\right\rangle\left\langle\psi_{\mathrm{u}}\left|\hat{\mu}^{\mathrm{i}}\right| \psi_{\mathrm{g}}\right\rangle\left\langle\psi_{\mathrm{g}}\left|\hat{\mu}^{\mathrm{j}}\right| \psi_{\mathrm{w}}\right\rangle\left\langle\psi_{\mathrm{w}}\left|\hat{\mu}^{\mathrm{k}}\right| \psi_{\mathrm{g}}\right\rangle}{\left(E_{\mathrm{gu}}-i \Gamma_{\mathrm{gu}}+\hbar \omega\right)\left(E_{\mathrm{gu}}-i \Gamma_{\mathrm{gu}}+\hbar \omega_{\mathrm{r}}\right)\left(E_{\mathrm{gw}}-i \Gamma_{\mathrm{gw}}+\hbar \omega_{\mathrm{p}}\right)}$

$\left.\left.+\frac{\left\langle\psi_{\mathrm{g}}\left|\hat{\mu}^{1}\right| \psi_{\mathrm{u}}\right\rangle\left\langle\psi_{\mathrm{u}}\left|\hat{\mu}^{\mathrm{i}}\right| \psi_{\mathrm{g}}\right\rangle\left\langle\psi_{\mathrm{g}}\left|\hat{\mu}^{\mathrm{j}}\right| \psi_{\mathrm{w}}\right\rangle\left\langle\psi_{\mathrm{w}}\left|\hat{\mu}^{\mathrm{k}}\right| \psi_{\mathrm{g}}\right\rangle}{\left(E_{\mathrm{gu}}-i \Gamma_{\mathrm{gu}}+\hbar \omega_{\mathrm{r}}\right)\left(E_{\mathrm{gw}}-i \Gamma_{\mathrm{gw}}-\hbar \omega_{\mathrm{q}}\right)\left(E_{\mathrm{gw}}-i \Gamma_{\mathrm{gw}}+\hbar \omega_{\mathrm{p}}\right)}\right]\right\}$

where the $\hbar \omega_{\mathrm{p}, \mathbf{q}, \mathrm{r}}$ terms denote the photon energies; $g$, the electronic ground state; $u, v$, and $w$, the electronic excited states; $E_{\text {gu }}$, the transition energy from state $g$ to state $u ; \hat{\mu}^{\mathrm{j}}$, the electric dipole moment operator along molecular axis $j ; \bar{\mu}^{\mathrm{j}}$, the transition dipole moment between states $u$ and $v$ only if $u$ and $v$ are different, or if $u$ and $v$ are the same then $\bar{\mu}^{\mathrm{j}}$ is the difference between the state dipole moments of state $u$ and the ground state; $i$, the imaginary unit; $\Gamma_{\mathrm{gu}}$, a damping factor for excited state $u$, which is related to the excited-state lifetime; and $I_{\mathrm{p}, \mathrm{q}, \mathrm{r}}$, the average over the terms obtained by simultaneous permutation of 
the incident photon frequency and the dipole moment operator. In molecular systems where only one excited state is strongly coupled to the ground state and the primary components of the state dipole moments and transition dipole moments are aligned along one molecular axis $x$, the full SOS expression can be simplified to an essential-state model with three terms, ${ }^{38-43}$ which in the static limit appears as:

$$
\begin{aligned}
& \gamma_{\mathrm{xxxx}} \propto \frac{\mu_{\mathrm{ge}}^{2} \Delta \mu_{\mathrm{eg}}^{2}}{E_{\mathrm{ge}}^{3}} \quad \mathbf{D} \\
& +\sum_{e^{\prime}} \frac{\mu_{\mathrm{ge}}^{2} \mu_{\mathrm{ee}^{\prime}}^{2}}{E_{\mathrm{ge}^{2}}^{2} E_{\mathrm{ge}^{\prime}}} \quad \mathbf{T} \\
& -\frac{\mu_{\text {ge }}^{4}}{E_{\text {ge }}^{3}} \quad \mathbf{N}
\end{aligned}
$$

The three terms are denoted $\mathbf{D}$ (for dipolar term, which is only non-zero in noncentrosymmetric systems), T (for two-photon term, which derives from the fact that in centrosymmetric systems this term involves transitions from the one-photon excited state $e$ to two-photon excited states $e^{\prime}$ ), and $\mathbf{N}$ (for negative term, due to the minus sign in front of the term).

In linear $\pi$-conjugated systems such as cyanines, ${ }^{27,44-46}$ donor-acceptor-substituted polyenes, ${ }^{26,27,44}$ and squaraines, ${ }^{28,31}$ molecular design principles describing the sign and magnitude of $\operatorname{Re}(\gamma)$ have been developed through understanding the relationship between the nature of the first several excited states and the geometric parameters related to bond-length alternation (BLA) along the $\pi$ backbone. However, this molecular understanding of $\operatorname{Re}(\gamma)$ has not yet been extended to higher-dimensional systems where the application of the essential-state model is not expected to be applicable.

Here, we present a new class of molecules with negative $\operatorname{Re}(\gamma)$ : tetraphenyl compounds $\mathrm{X}\left(\mathrm{C}_{6} \mathrm{H}_{5}\right)_{4}$, $\mathrm{X}=\mathrm{B}^{-}, \mathrm{C}, \mathrm{N}^{+}$, and $\mathrm{P}^{+}($ 

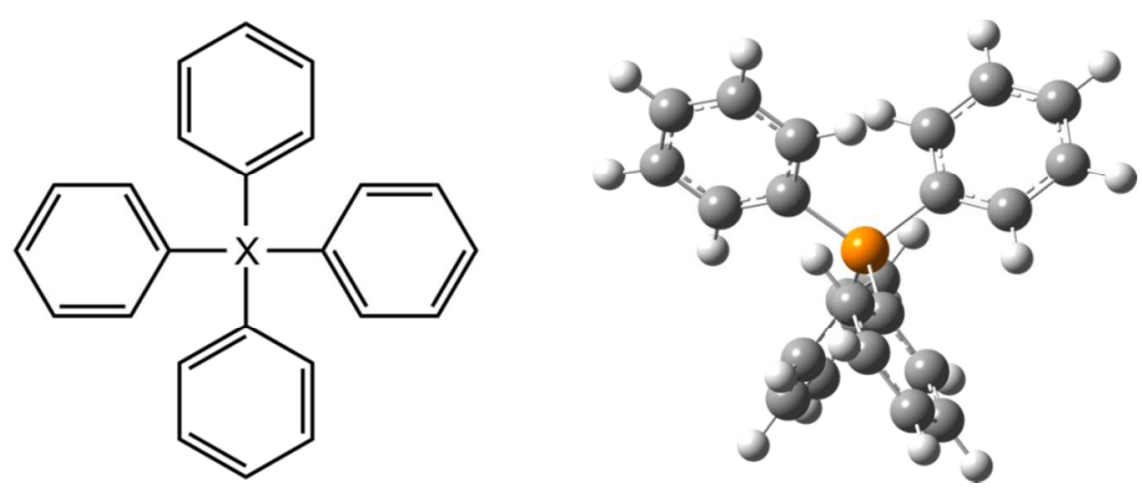

Figure 1). We first describe the geometric and electronic structures of these molecules, which have threedimensional $\pi$ systems and do not correspond with the geometric parameters typically used to understand the sign of $\operatorname{Re}(\gamma)$ in linear conjugated systems. We then discuss the excited-state properties and NLO properties and demonstrate that the sign and magnitude of $\operatorname{Re}(\gamma)$ can be understood only in terms of the properties of a band of excited states in the context of the full SOS model. Finally, we present experimental confirmation that $\operatorname{Re}(\gamma)$ at the static limit is indeed negative in these systems.

\section{Figure 1. General chemical structure of the $\mathrm{XPh}_{4}$ series $\left(X=\mathrm{B}^{-}, \mathrm{C}, \mathrm{N}^{+}, \mathrm{P}^{+}\right)$and sketch of the geometric structure of $\mathrm{PPh}_{4}{ }^{+}$.}

\section{Methods}

\subsection{Computational Methodology}

The geometric structures were optimized via density functional theory (DFT) using the $\omega$ B97XD functional $^{47,48}$ and cc-pVDZ basis set $^{49}$ as implemented in the Gaussian 09 (Rev. B.01) suite of programs. ${ }^{50}$ All geometry minima were confirmed by the absence of imaginary frequencies. As the molecules have $\mathrm{S}_{4}$ symmetry, the $z$ axis was defined as the axis of symmetry. The excited-state properties were then evaluated using a configuration interaction (CI) approach using single-electron excitations 
(SCI) with the INDO Hamiltonian; ${ }^{27,51,52}$ this approach has previously provided excellent agreement with the experimental NLO properties of $\pi$-conjugated systems. ${ }^{53,54}$ The CI active space included all singleelectron excitations within the 25 highest-lying occupied molecular orbitals (MOs) and 25 lowest-lying unoccupied MOs. Although double-electron excitations are usually critical to accurately compute the lowlying excited state properties of $\pi$-conjugated systems, ${ }^{51,53,55-57}$ test calculations incorporating doubleelectron excitations through SDCI (single and double CI) or MRDCI (multi-reference determinant CI) schemes show that the first 40-60 excited states in these systems contain negligible double-excitation character; hence, excitations with such character can be safely neglected through use of the SCI approach.

The NLO properties were computed using the sum-over-states approach (power series expansion). The total static $\operatorname{Re}(\gamma)$ and $\operatorname{Im}(\gamma)$ evaluated by summing over 100 states. Decomposition of the contributions from each of the excited states was achieved by calculating the static $\gamma$ value separately for each state $u$ in Eqn. 2. The static $\gamma$ was also decomposed into contributions from the $\mathbf{D}, \mathbf{T}$, and $\mathbf{N}$ terms; in doing so, the first summation in the full SOS expression, comprises the $\mathbf{D}(u=v=w)$ and $\mathbf{T}(u \neq v$ and/or $v \neq w)$ terms and the second summation comprises the $\mathbf{N}$ term. The orientationally averaged $\operatorname{Re}(\gamma)$ is computed from the tensor components as:

$$
\begin{aligned}
\gamma_{\mathrm{avg}} & =\frac{1}{5}\left(\gamma_{\mathrm{xxxx}}+\gamma_{\mathrm{yyyy}}+\gamma_{\mathrm{zzzz}}\right) \\
& +\frac{1}{15}\left(\gamma_{\mathrm{xxyy}}+\gamma_{\mathrm{yyxx}}+\gamma_{\mathrm{xyyx}}+\gamma_{\mathrm{xyxy}}+\gamma_{\mathrm{yxyx}}+\gamma_{\mathrm{yxxy}}\right. \\
& +\gamma_{\mathrm{xxzz}}+\gamma_{\mathrm{zzxx}}+\gamma_{\mathrm{xzzx}}+\gamma_{\mathrm{xzxz}}+\gamma_{\mathrm{zxzx}}+\gamma_{\mathrm{zxxz}} \\
& \left.+\gamma_{\mathrm{zzyy}}+\gamma_{\mathrm{yyzz}}+\gamma_{\mathrm{zyyz}}+\gamma_{\mathrm{zyzy}}+\gamma_{\mathrm{yzyz}}+\gamma_{\mathrm{yzzy}}\right)
\end{aligned}
$$

\subsection{Experimental Methodology}


The linear absorption of $\mathrm{PPh}_{4}{ }^{+}$dissolved in spectroscopic grade chloroform was measured using a Varian Cary 500 spectrophotometer. To study the possible effects of aggregation, we prepared solutions ranging from concentrations of $22 \mu \mathrm{M}$ up to $13 \mathrm{mM}$, and measured their absorption spectra in quartz cuvettes with thicknesses varying from $1 \mathrm{~cm}$ to $0.1 \mathrm{~mm}$, respectively.

To measure the $\operatorname{Re}(\gamma)$ far from the one-photon absorption resonance, we used the recently developed dualarm (DA) Z-scan technique, ${ }^{58}$ an extension of the conventional Z-scan method. ${ }^{59}$ The DA Z-scan technique allows for the simultaneous subtraction of the solvent nonlinear signal from the solution. For simultaneous subtraction, the two arms are identically matched in terms of the irradiance parameters, i.e. pulse energy, beam waist, pulse width, and sample positioning, and as a result the correlated noise from the excitation source is cancelled. This leaves only the nonlinear signal due to the solute with a large increase in the signal-to-noise ratio. This significantly improves our ability to measure the $\operatorname{Re}(\gamma)$ when approaching the static limit where the nonlinear refraction is small. In this regime, the typically large signal from the solvent can mask that due to the solute. Furthermore, the $\operatorname{Im}(\gamma)$, corresponding to nonlinear optical losses, is essentially zero far from resonance, thus the DA Z-scan signals presented in the subsequent section are solely attributed to $\operatorname{Re}(\gamma)$.

\section{Results and Discussion}

\subsection{Computational Results}

\subsubsection{Ground-state properties}

We start by discussing the geometric and electronic structures of the series of tetraphenyl compounds $\mathrm{X}\left(\mathrm{C}_{6} \mathrm{H}_{5}\right)_{4}$, where $\mathrm{X}=\mathrm{B}^{-}, \mathrm{C}, \mathrm{N}^{+}$, and $\mathrm{P}^{+}$. All of these systems have energetic minima in geometries with $\mathrm{S}_{4}$ symmetry. The bond lengths within the phenyl rings are similar across the series, and the rings maintain the aromatic character associated with isolated benzene rings. This can be seen through analysis of the quinoidal-benzene character (QBC), defined as $\sum_{i}\left(\mid r_{i}-1.4 \AA\right)$ ) where $r_{i}$ is the length of each C-C bond $i$ 
in the phenyl ring. ${ }^{28}$ The QBC values for these compounds are all very small $(<0.05 \AA$; we note that for squaraines, the crossover point from aromatic-like structures with positive $\operatorname{Re}(\gamma)$ to quinoid-like structures with negative $\operatorname{Re}(\gamma)$ was computed to be on the order of $0.17 \AA) .{ }^{28}$ This indicates that the phenyl rings largely retain their aromatic character; the quinoidal form has a minimal contribution to the ring conjugation pattern. It is useful to recall at this stage that the negative $\operatorname{Re}(\gamma)$ in cyanines and squaraines can be attributed to the molecule being in an intermediate geometry between two resonance forms, where the ground state is highly polarizable, more so than any low-lying excited state. In the $\mathrm{XPh}_{4}$ series, the nature of the molecular geometries suggests that the negative $\operatorname{Re}(\gamma)$ cannot be attributed to the same cause.

The frontier molecular orbitals (MOs) in the $\mathrm{XPh}_{4}$ series are $\pi$-orbitals. The eight highest occupied MOs are composed of linear combinations of the two highest occupied orbitals of each of the four phenyl rings; similarly, the eight lowest unoccupied MOs are composed of linear combinations of the two lowest unoccupied orbitals of each phenyl ring (representations of the MOs are provided in the SI). In all members of the series, the HOMO is doubly degenerate, whereas the LUMO is non-degenerate. The nondegenerate MOs are fully delocalized across all four phenyl rings (in the degenerate MO pairs, the total electron density of the two orbitals can also be seen as distributed evenly across all four phenyl rings). Because the coupling between the orbitals on different phenyl rings is small, the energetic spacing within each of these sets of eight MOs is small enough to effectively form bands of occupied and unoccupied $\pi$ orbitals. Such an electronic structure is markedly different from that seen in most linear $\pi$-conjugated systems, which typically have significant energetic gaps separating frontier MOs. Since the central atom has little contribution to the frontier MOs, the HOMO-LUMO gap is relatively unaffected by changes to the central atom and its calculated value varies only between $9.44 \mathrm{eV}\left(\mathrm{B}^{-}\right)$and $9.61 \mathrm{eV}\left(\mathrm{N}^{+}\right)$within the series. 
Figure 2. Molecular orbital energies (HOMO-7 through LUMO+7) at the INDO level in the $\mathrm{XPh}_{4}$ series.

\subsubsection{Excited-state properties}

We now turn to the excited-state energies, configuration-interaction compositions, and state-dipole and transition-dipole moments. This discussion will center on how the first several frontier MOs determine the low-lying excited-state properties. As the MOs and excited-state properties do not change substantially among the members of this series, we have chosen here to focus on $\mathrm{PPh}_{4}{ }^{+}$. Because the frontier MOs have the band-like energetic spacing discussed in the previous Section, the compositions of the $\mathrm{XPh}_{4}$ excited states are much more complex than those of typical linear $\pi$-conjugated systems. The first several excited states have little one-photon or two-photon activity; instead, the optical and NLO properties are dependent on many higher-lying states, as will be discussed in more detail in the following paragraphs. Understanding the general trends in the energies and transition dipole moments among many excited states is critical to understanding the molecular optical and NLO properties. 
Because of the relatively small energetic gaps among the first eight HOMOs and the first eight LUMOs, the single-electron excitations within this range of molecular orbitals are all relatively similar in energy (a total of 64 single excitations) and are energetically well-separated from any other excitations within the $\pi$ system. The first 40-60 excited states are composed of linear combinations of these low-lying single excitations, with negligibly small contributions from higher-energy excitations (Table 1). The first excited state has an energy of $4.64 \mathrm{eV}$; however, we focus here on the higher-lying excited states within this band that are more strongly coupled to the ground state. Each excited state is composed of a linear combination of many single excitations; in only a few excited states does any one excitation compose more than $30 \%$ of the state electronic configuration $(\mathrm{CI}$ coefficient $=0.55)$.

Table 1. State energies, key dipole moment parameters, and CI composition of excited states of $\mathrm{PPh}_{4}{ }^{+}$; all excited states within the first 100 states with $\mu_{\mathrm{ge}}>4$ Debye are listed, and $x, y$, and $z$ denote the molecular axes, where $z$ is the axis of symmetry.

\begin{tabular}{|c|c|c|c|c|}
\hline State & $\begin{array}{c}\text { Energy } \\
(\mathrm{eV})\end{array}$ & $\begin{array}{c}\Delta \mu_{\mathrm{eg}} \\
\text { (Debye) }\end{array}$ & $\mu_{\mathrm{ge}}$ (Debye) & CI composition \\
\hline 17 & 6.44 & $-0.57 z$ & $-5.47 x-0.28 y$ & $\begin{aligned}-0.32 \mid H-4 \rightarrow & L+4\rangle-0.32|H-4 \rightarrow L+5\rangle \\
& +0.43|H-1 \rightarrow L\rangle \\
& -0.32|H \rightarrow L+1\rangle\end{aligned}$ \\
\hline 18 & 6.44 & $0.57 z$ & $0.28 x-5.47 y$ & $\begin{aligned}-0.32 \mid H-4 \rightarrow & L+4\rangle-0.32|H-4 \rightarrow L+5\rangle \\
& +0.32|H-1 \rightarrow L+1\rangle \\
& +0.43|H \rightarrow L\rangle\end{aligned}$ \\
\hline 19 & 6.45 & 0 & $-6.80 z$ & $\begin{aligned}+0.34 \mid H-6 \rightarrow & L+5\rangle-0.33|H-4 \rightarrow L+7\rangle \\
& +0.33|H-3 \rightarrow L+6\rangle \\
& -0.34|H-2 \rightarrow L+4\rangle\end{aligned}$ \\
\hline 20 & 6.47 & $-6.27 z$ & $-5.94 x-3.93 y$ & $-0.53|H-1 \rightarrow L\rangle-0.42|H-1 \rightarrow L+1\rangle$ \\
\hline 21 & 6.47 & $6.27 z$ & $-3.93 x+5.94 y$ & $+0.53|H \rightarrow L\rangle-0.42|H \rightarrow L+1\rangle$ \\
\hline 31 & 6.82 & 0 & $-5.40 z$ & $\begin{aligned}+0.50 \mid H-5 \rightarrow & L\rangle-0.36|H-4 \rightarrow L+3\rangle \\
& +0.36|H-3 \rightarrow L+2\rangle\end{aligned}$ \\
\hline
\end{tabular}

We note that the excited states exhibit symmetry-breaking if their geometries are allowed to relax. In particular, at the CIS level, the $\mathrm{C}_{1}$-optimized structure of the first excited state is stabilized by $0.1 \mathrm{eV}$ as 
compared to the $\mathrm{S}_{4}$-optimized geometry. The geometric changes in the $\mathrm{C}_{1}$ geometry suggest that the excitation becomes localized primarily on one phenyl ring; one P-C bond is shortened by $0.04 \AA$ and the $\mathrm{C}-\mathrm{C}$ bonds in that phenyl ring are lengthened by $0.03 \AA$ relative to the other three phenyl rings. However, the electronic NLO processes occur substantially faster than geometric relaxations and involve the excited states as virtual states. Thus, here, we focus solely on the excited-state properties in the $\mathrm{S}_{4}$ ground-state geometry.

To understand the state dipole moments and the transition dipole moments, we first consider the contributions of the pure single-electron excitations, then evaluate the effect of the linear combinations of excitations in the excited states. We turn first to the state dipole moments. In the ground state, the $\mathrm{S}_{4}$ molecular symmetry implies that the dipole moment $\mu_{\mathrm{g}}$ is zero; ${ }^{60}$ thus, the difference $\Delta \mu_{\mathrm{eg}}$ between the excited-state and ground-state dipole moments is equal to the excited-state dipole moment $\mu_{\mathrm{e}}$. The excited-state dipole moments depend on the electron distribution, related to the molecular orbital spatial distributions. Since most of the molecular orbitals are symmetrically distributed across the molecule, the single excitations between these orbitals do not change the molecular dipole moment. However, in transitions involving molecular orbitals in degenerate pairs, the two transitions involving each orbital in the pair will have state dipole moments along the molecular $z$ axis that are equal in magnitude but opposite in sign.

The excited-state dipole moments $\mu_{\mathrm{e}}$ can be considered as weighted sums of the changes in the state dipole moment due to each component electronic configuration. In the excited states with no contribution from excitations involving degenerate orbitals, all excitations have contributions of zero to $\mu_{\mathrm{e}}$, so $\mu_{\mathrm{e}}=0$. If the contributions from pairs of degenerate excitations are equal in magnitude, the contributions to the state dipole moment cancel and $\mu_{\mathrm{e}}=0$. However, if the contributions from degenerate excitations are not 
equal, as happens in pairs of degenerate excited states, $\mu_{\mathrm{e}}$ can be as large as 6 Debye; within each pair of degenerate states, the two $\mu_{\mathrm{e}}$ values are equal in magnitude but opposite in sign.

For each excited state $e$, the transition dipole moment $\mu_{\text {ge }}$ to the ground state is likewise composed of a linear combination of contributions from each component excitation. The upper 8 HOMOs are composed of linear combinations of the phenyl orbitals that have one node within each phenyl ring, whereas the lower 8 LUMOs are composed of linear combinations of the phenyl orbitals that have two nodes within each phenyl ring. In each excitation from one of the first 8 HOMOs to one of the first 8 LUMOs, the differing number of nodes within each phenyl ring implies that each ring has some atoms where the transition density has a positive sign and some where the transition density has a negative sign. The cancellation of positive and negative transition densities within each phenyl ring limits the magnitude of the orbital transition dipole moments. The orbital components of the transition dipole moments are therefore relatively small, ranging from 0-4 Debye, ${ }^{61}$ and may be aligned either along the molecular $z$ axis or in the $x y$ plane.

Since the low-lying excited states are composed of single excitations, the transitions from the ground state to each CI component in each excited state all involve electronic configurations that are different by one orbital. Thus, the transition dipole moments $\mu_{\mathrm{ge}}$ can be computed as linear combinations of the transition dipole moments of all component excitations. Depending on the signs of the CI coefficients and the orbital transition dipole moments, the components can combine additively or subtractively. Several excited states have significant $\mu_{\text {ge }}$ (Table 1); as will be detailed later, these states give the most significant contributions to the linear and nonlinear optical properties. Notably, there are states with large $\mu_{\text {ge }}$ both along the molecular $z$ axis and in the $x y$ plane. This is different from typical linear $\pi$-conjugated molecules, which commonly have only one low-lying excited state that is significantly coupled to the ground state. $^{27}$ 
The transition dipole moments $\mu_{\mathrm{ee}}$, between excited states involve linear combinations of transitions between singly-excited electron configurations. Since only transitions between configurations that differ by no more than one orbital can have non-zero contributions to the transition dipole moment, only configuration pairs in which the excitations involve either the same occupied orbital or the same unoccupied orbital can contribute to $\mu_{\mathrm{ee}}$. An allowed transition between two singly excited configurations therefore involves a one-electron transition either within the occupied manifold or within the unoccupied manifold. The transitions between two HOMOs or between two LUMOs can have much larger orbital transition dipole moments than the transitions between one HOMO and one LUMO because the transition densities within each ring can all contribute with the same sign to the transition dipole moment. The orbital components of the transition dipole moments range from 0 to 9 Debye, up to a factor of two larger than for the HOMO-LUMO transitions; again, these components can be aligned either along the $z$ axis or in the $x y$ plane.

Even though the orbital contributions to $\mu_{\mathrm{ee}}$ are large, the significant mixing of excitations in each excited state limits the magnitude of $\mu_{\mathrm{ee}}$. Most pairs of excitations differ by two orbitals and have no contribution to $\mu_{\mathrm{ee}}$. In configuration pairs that do contribute to $\mu_{\mathrm{ee}}$, the orbital component is multiplied by two relatively small CI coefficients (as noted previously, typically $<0.55$ ), so only infrequently does any one configuration pair contribute more than 1 Debye to $\mu_{\mathrm{ee}}$. In transitions with multiple configuration pairs contributing to $\mu_{\mathrm{ee}}$, the terms may contribute additively or subtractively. Importantly, the excited states that are strongly coupled to the ground state have few large transition dipole moments to other excited states ( 
Table 2). This weak coupling between excited states implies that there is relatively little 2PA in these systems; as will be described in the following Section, this also has important implications in determining the sign of $\operatorname{Re}(\gamma)$. 
Table 2. Transition dipole moments between excited states in $\mathrm{PPh}_{4}{ }^{+}$; all transition dipole moments greater than 4 Debye from the states listed in Table 1 to other excited states are listed.

\begin{tabular}{|c|c|}
\hline Transition & $\boldsymbol{\mu}_{\mathbf{e e}^{\prime}}$ (Debye) \\
\hline $19 \rightarrow 25$ & $4.94 z$ \\
\hline $20 \rightarrow 23$ & $5.50 z$ \\
\hline $20 \rightarrow 25$ & $2.76 x+3.44 y$ \\
\hline $21 \rightarrow 24$ & $5.50 z$ \\
\hline $21 \rightarrow 25$ & $3.44 x-2.76 y$ \\
\hline
\end{tabular}

\subsubsection{Nonlinear optical properties}

We can understand the negative sign of $\operatorname{Re}(\gamma)$ in terms of the excited-state energies, state dipole moments, and transition dipole moments. As will be discussed in the following section, the magnitude and sign of the computed $\operatorname{Re}(\gamma)$ are in good agreement with the experimental results. Here, we focus on $\operatorname{Re}(\gamma)$ at the static (zero-frequency) limit. As mentioned previously, because there are several excited states with large coupling to the ground state along different molecular axes, the commonly-used essential-state model cannot be applied to these systems. Instead, we discuss the NLO properties in terms of the full SOS expression (Eqn. 2). Although this expression might at first look complex, we recall that each term simply consists of a product of four transition-dipole and/or state-dipole terms in the numerator and a product of three state-energy terms in the denominator.

To provide insight into the origins of the NLO properties, we decompose $\gamma$ into contributions from each one-photon state $u$ in Eqn. 2. As $\operatorname{Im}(\gamma)$ is negligibly small at the static limit, we focus solely on $\operatorname{Re}(\gamma)$. The $\operatorname{Re}(\gamma)$ values presented here are orientationally averaged as described in Eqn. 4; unlike in linear $\pi$ conjugated systems, the total $\operatorname{Re}(\gamma)$ is nearly isotropic in these systems because there are large $\mu_{\text {ge }}$ terms along all three molecular axes. As shown in 
Table $3, \operatorname{Re}(\gamma)$ contains significant contributions from a number of excited states, particularly those that are strongly coupled to the ground state. 
Table 3. $\operatorname{Re}(\gamma)$ and its major state components and term decomposition of $\mathrm{PPh}_{4}{ }^{+}\left(\mathrm{x} 10^{-36}\right.$ esu). All states with total contributions $>2 \times 10^{-36}$ esu are included.

\begin{tabular}{|c|c|c|c|c|}
\hline State & Total & D & T & N \\
\hline 17 & -3.3 & 0.0 & 0.7 & -4.0 \\
\hline 18 & -3.3 & 0.0 & 0.7 & -4.0 \\
\hline 19 & -5.4 & 0.0 & 0.7 & -6.1 \\
\hline 20 & -5.9 & 0.5 & 0.2 & -6.6 \\
\hline 21 & -5.9 & 0.5 & 0.2 & -6.6 \\
\hline 31 & -3.2 & 0.0 & 0.3 & -3.5 \\
\hline Total & -39.8 & 1.1 & 2.1 & -43.0 \\
\hline
\end{tabular}

We also consider the contributions to $\operatorname{Re}(\gamma)$ from each of the three terms as decomposed in the essentialstate model. Because the excited states with significant contributions to $\operatorname{Re}(\gamma)$ all fall within a relatively narrow energy window, the denominators of all terms are similar in magnitude and we can focus on the numerators. The second summation in the full SOS expression (corresponding to the $\mathbf{N}$ term in the essential-state model) contains a product of four $\mu_{\text {ge }}$ terms in the numerator. Although the essential-state model simplifies this term to the form $-\mu_{\mathrm{ge}}^{4} / E_{\mathrm{ge}}^{3}$, the full SOS expression also includes terms of the form $-\left(\mu_{\mathrm{gu}}^{2} \mu_{\mathrm{gw}}^{2}\right) /\left(E_{\mathrm{gu}}^{2} E_{\mathrm{gw}}\right)$ where $u$ and $w$ are both excited states. When multiple excited states are significantly coupled to the ground state, these cross-terms have significant contributions to $\operatorname{Re}(\gamma)$. Because the $\mathrm{XPh}_{4}$ systems have several excited states that are coupled to the ground state, these terms have a significant negative contribution to $\operatorname{Re}(\gamma)$.

The first summation in the SOS expression has an additional dependence on the transition dipole moments $\mu_{\mathrm{ee}}$, between excited states ( $\mathbf{T}$ term) and the state dipole moments $\Delta \mu_{\mathrm{eg}}(\mathbf{D}$ term). Since the states with significant coupling to the ground state have small $\Delta \mu_{\text {eg }}$ and few large couplings to other excited states, these terms are at least an order of magnitude smaller than the $\mathbf{N}$ term. Because the $\mathbf{N}$ term has the dominant contribution in the $\operatorname{SOS}$ expression, $\operatorname{Re}(\gamma)$ is negative. This behavior is substantially different 
from that typically seen in $\pi$-conjugated systems, where large couplings between the excited states cause the $\mathbf{T}$ term to dominate and $\operatorname{Re}(\gamma)$ to be positive. ${ }^{62-65}$

Although our discussion has privileged $\mathrm{PPh}_{4}{ }^{+}$, the linear and nonlinear optical properties of all four members of the $\mathrm{XPh}_{4}$ series are largely similar. The energetic spacing of the first several frontier MOs is hardly affected by the identity of the central atom, so a similar mixing of many excitations in the lowlying excited states is observed. The four systems have similar NLO properties, with many states contributing significantly to $\operatorname{Re}(\gamma)$. The NLO properties are dependent on the spatial distribution and band-like energetic spacing of the first several frontier MOs, not on the identity of the central atom.

Table 4. $\operatorname{Re}(\gamma)$ and its term decomposition for the $\mathrm{XPh}_{4}$ series

\begin{tabular}{|c|c|c|c|c|}
\hline \multirow{2}{*}{$\begin{array}{c}\text { Central } \\
\text { Atom }\end{array}$} & \multicolumn{4}{|c|}{$\operatorname{Re}(\boldsymbol{\gamma})\left(\mathbf{x} \mathbf{1 0}^{-36} \mathbf{e s u}\right)$} \\
\cline { 2 - 5 } & Total & $\mathbf{D}$ & $\mathbf{T}$ & $\mathbf{N}$ \\
\hline $\mathrm{B}^{-}$ & -44.2 & 0.7 & 5.8 & -50.6 \\
\hline $\mathrm{C}$ & -42.0 & 1.0 & 6.8 & -49.8 \\
\hline $\mathrm{N}^{+}$ & -39.3 & 1.7 & 6.2 & -47.2 \\
\hline $\mathrm{P}^{+}$ & -39.8 & 1.1 & 2.1 & -43.0 \\
\hline
\end{tabular}

\subsection{Experimental Results}

\subsubsection{Linear Absorption Spectroscopy}

The linear absorption of $\mathrm{PPh}_{4}{ }^{+}$dissolved in spectroscopic grade chloroform (

Figure 3) exhibits a broad, multi-peak absorption band between $250 \mathrm{~nm}$ and $280 \mathrm{~nm}(5.0-4.4 \mathrm{eV})$, with a maximum molar absorptivity of $0.42 \pm 0.04 \times 10^{4} \mathrm{~cm}^{-1} \mathrm{M}^{-1}$ at $276 \mathrm{~nm}(4.49 \mathrm{eV})$. The molar absorptivity increases drastically at wavelengths shorter than $250 \mathrm{~nm}(5.0 \mathrm{eV})$; however, the absorption above $220 \mathrm{~nm}$ 
$(5.6 \mathrm{eV})$ is not resolvable due to the high absorption of the solvent. Hence, the spectrophotometer cannot accurately subtract the transmission of the solvent from the solution at these wavelengths. The shape of the experimental absorption peak is consistent with the computed excited-state properties, which predict weak absorption into the first several excited states and much stronger absorption into higher-lying excited states.

The prepared solutions with concentrations ranging from $22 \mu \mathrm{M}$ to $\sim 91 \mathrm{mM}$ in cuvette thicknesses from $10 \mu \mathrm{m}$ to $1 \mathrm{~cm}$ showed no significant difference in the absorption shape in the $250-280 \mathrm{~nm}$ range, which indicates that there is no significant aggregation of the solute in this concentration range.

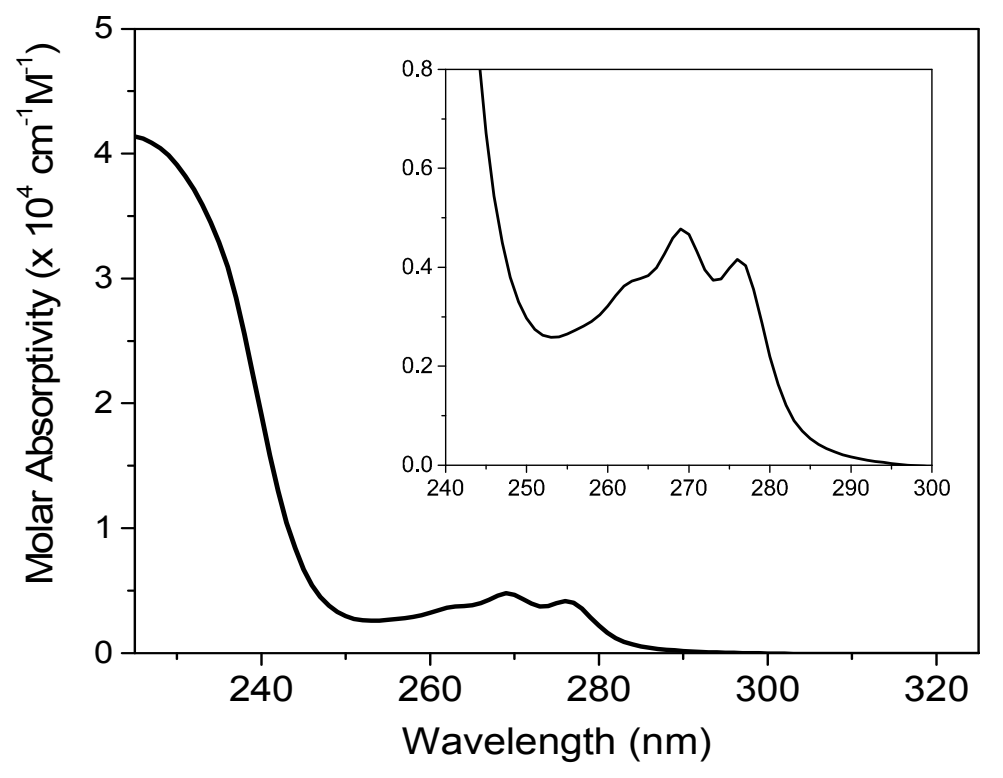

Figure 3. Molar absorptivity spectrum of $\mathrm{PPh}_{4}{ }^{+}$dissolved in chloroform. The inset, having the same axis titles as the main figure, shows the molar absorptivity with the vertical and horizontal axes expanded. 


\subsubsection{Nonlinear Spectroscopy}

To perform the DA Z-scan measurements outlined in Section 2.2., a Ti:Sapphire amplified laser (ClarkMXR CPA 2110) producing $1 \mathrm{~mJ}, \sim 150 \mathrm{fs}(\mathrm{FWHM})$ pulses at a $1 \mathrm{kHz}$ repetition rate pumps an optical parametric generator/amplifier (Light Conversion, Ltd., TOPAS-C) to generate pulses at 1300nm for Zscans. To characterize the minimum spot size and pulse-width of our input pulses, Z-scans were performed on two reference materials: fused silica and a bulk sample of GaAs. The Z-scan of fused silica gave an $n_{2}$ of $0.27 \pm 0.05 \times 10^{-15} \mathrm{~cm}^{2} / \mathrm{W}$ which is in good agreement with literature values ${ }^{66}$ and the Zscan of GaAs gave a $2 \mathrm{PA}$ coefficient, $\alpha_{2}$, of $27 \pm 5 \mathrm{~cm} / \mathrm{GW}$ also in good agreement with literature values and a two-band theoretical model. ${ }^{67,68}$ These Z-scans of the reference materials gave the minimum spot size and pulse width of $22.5 \mu \mathrm{m}\left(\mathrm{HW} 1 / \mathrm{e}^{2} \mathrm{M}\right)$ and $130 \mathrm{fs}(\mathrm{FWHM})$, respectively.

To measure the signals from the DA Z-scan, a $91 \mathrm{mM}$ concentration of $\mathrm{PPh}_{4}{ }^{+}$in chloroform was placed in a $1 \mathrm{~mm}$ thick quartz cuvette in one arm of the DA Z-scan while the pure solvent was placed in a closely matched $1 \mathrm{~mm}$ thick quartz cuvette in the other arm. Figure 4(a) shows the Z-scans of $\mathrm{PPh}_{4}{ }^{+}$at a wavelength of $1300 \mathrm{~nm}$ using 3 different input pulse energies. At this wavelength, the excitation photon energy is $\sim 5 \times$ below its linear absorption edge; hence, we can assume that the value of $\operatorname{Re}(\gamma)$ measured at this wavelength is close to its static (zero-frequency) limit. There was no observable Z-scan signal corresponding to nonlinear absorption, which suggests a negligible $\operatorname{Im}(\gamma)$. The $\mathrm{n}_{2}$ coefficients are relatively constant over the range of input irradiances (see Figure 4(b)), indicating that there are no higher-order and/or cascaded NLR processes occurring. The error bars are deduced from the uncertainty in the pulse irradiance as well as the fitting errors to each scan. Therefore, we measure an $n_{2}$ of the solute at $1300 \mathrm{~nm}$ of $-0.11 \pm 0.03 \times 10^{-15} \mathrm{~cm}^{2} / \mathrm{W}$ corresponding to an averaged molecular $\operatorname{Re}(\gamma)=-31 \pm$ $8 \times 10^{-36}$ esu (see Eqn. 4). This value of $\operatorname{Re}(\gamma)$ is in very good agreement with the theoretical results shown in Table 4, both in terms of sign and magnitude. It must be borne in mind, however, that such a value is several orders of magnitude smaller than values recently reported for selenopyrylium 
polymethines. ${ }^{12,69}$ Note that despite $\operatorname{Re}(\gamma)$ of $\mathrm{PPh}_{4}{ }^{+}$being $\sim 20 \mathrm{x}$ larger than the averaged $\operatorname{Re}(\gamma)$ of the solvent chloroform molecule, given that the concentration of the neat liquid is $12.47 \mathrm{M}$, the measured NLR signal from $\mathrm{PPh}_{4}{ }^{+}$is $\sim 7$ times smaller than that of neat chloroform and a factor of $\sim 2$ times smaller than that due to the quartz cuvette walls. Thus, the use of DA Z-scan was essential for these measurements.
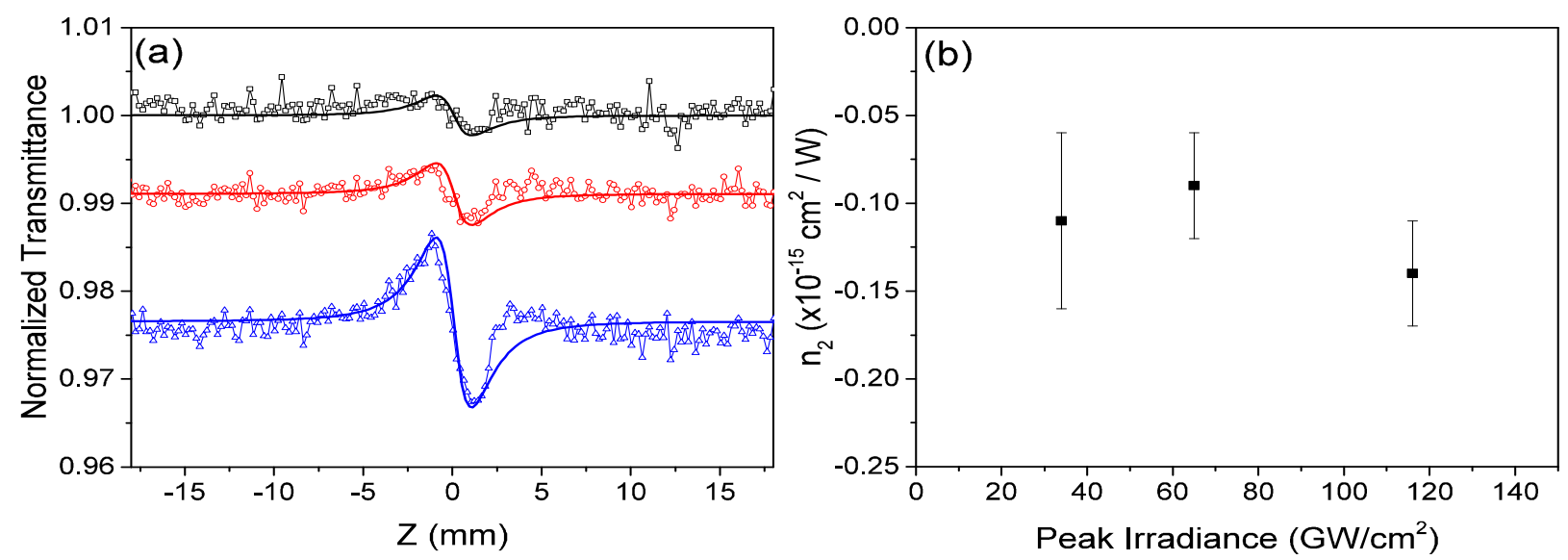

Figure 4. (a) DA Z-scans of a $91 \mathrm{mM}$ concentration of $\mathrm{PPh}_{4}{ }^{+}$at the laser wavelength of $1300 \mathrm{~nm}$ using different input pulse energies. (b) The values of the Z-scan fits with respect to the input peak irradiance. The open symbols in (a) represent the data while the solid lines represent the fits to the data.

\section{Conclusions}

Although a negative $\operatorname{Re}(\gamma)$ is advantageous for device applications, very few classes of molecules studied to date have negative $\operatorname{Re}(\gamma)$. Here, we present experimental evidence that molecules of the form $\mathrm{XPh}_{4}$ have negative $\operatorname{Re}(\gamma)$ and provide a theoretical understanding of the NLO behavior in terms of the molecular orbitals and excited states. Experimentally, the accurate determination of nonlinearities far from resonances of molecules in solution has been problematic as the solvent nonlinearity typically 
dominates. The recent development of nonlinear differential techniques such as the dual-arm Z-scan have made it possible to overcome such limitations.

Unlike in typical linear conjugated systems, the NLO properties of the $\mathrm{XPh}_{4}$ series depend on a band of several tens of low-lying excited states, of which several excited states are significantly coupled to the ground state. Since the $\pi$ systems here are inherently three-dimensional, $\operatorname{Re}(\gamma)$ is nearly isotropic, in contrast with typical linear $\pi$-conjugated systems that have significant nonlinearity primarily along a single molecular axis. Because of the complexity of the excited-state properties, the NLO properties must be understood in terms of the full SOS expression and cannot be simplified in terms of the commonly used essential-state model. Although the magnitude of $\operatorname{Re}(\gamma)$ is relatively small, the discovery of a new molecular architecture offering the potential for a negative $\operatorname{Re}(\gamma)$ provides new molecular design approaches to achieve a large negative $\operatorname{Re}(\gamma)$ for device applications.

\section{Acknowledgements}

We thank Drs. Joel Hales, Seth Marder, and Joseph Perry for stimulating discussions. The work at Georgia Tech was supported by the AFOSR MURI program (FA9550-10-1-0558) within the Center for Organic Materials for All-Optical Switching (COMAS). CR thanks the University of Kentucky Vice President for Research for start-up research funds. JLB acknowledges generous support from King Abdullah University of Science and Technology. EVS and DJH thank the National Science Foundation (NSF) (ECCS-1202471, ECCS-1229563) for support.

\section{Supporting Information}

Additional details are provided about the computations of atomic charges, molecular orbitals, decomposition of transition dipole moments into orbital components, and state contributions to $\operatorname{Re}(\gamma)$. This material is available free of charge via the Internet at http://pubs.acs.org. 


\section{References}

(1) Kim, T.-D.; Kang, J.-W.; Luo, J.; Jang, S.-H.; Ka, J.-W.; Tucker, N.; Benedict, J. B.; Dalton, L.; Gray, T.; Overney, R. M.; Park, D. H.; Herman, W. N.; Jen, A. K. Y. J. Am. Chem. Soc. 2007, $129,488$.

(2) Dalton, L. R.; Benight, S. J.; Johnson, L. E.; Knorr, D. B.; Kosilkin, I.; Eichinger, B. E.; Robinson, B. H.; Jen, A. K. Y.; Overney, R. M. Chem. Mater. 2011, 23, 430.

(3) Dalton, L. R.; Sullivan, P. A.; Bale, D. H. Chem. Rev. 2010, 110, 25.

(4) Kwon, O. P.; Kwon, S.-J.; Jazbinsek, M.; Seo, J.-Y.; Kim, J.-T.; Seo, J.-I.; Lee, Y. S.; Yun, H.; Günter, P. Chem. Mater. 2011, 23, 239.

(5) Campagnola, P. J.; Loew, L. M. Nature Biotechn. 2003, 21, 1356.

(6) Zipfel, W. R.; Williams, R. M.; Christie, R.; Nikitin, A. Y.; Hyman, B. T.; Webb, W. W. Proc. Natl. Acad. Sci. USA 2003, 100, 7075.

(7) Albota, M. A.; Beljonne, D.; Brédas, J. L.; Ehrlich, J. E.; Fu, J. Y.; Heikal, A. A.; Hess, S.; Kogej, T.; Levin, M. D.; Marder, S. R.; McCord-Maughon, D.; Perry, J. W.; Röckel, H.; Rumi, M.; Subramaniam, G.; Webb, W. W.; Wu, X. L.; Xu, C. Science 1998, 281, 1653.

(8) Pawlicki, M.; Collins, H. A.; Denning, R. G.; Anderson, H. L. Angew. Chem. Int. Ed. $\mathbf{2 0 0 9}, 48,3244$.

(9) He, G. S.; Tan, L.-S.; Zheng, Q.; Prasad, P. N. Chem. Rev. 2008, 108, 1245.

(10) Spangler, C. W. J. Mater. Chem. 1999, 9, 2013.

(11) Hales, J. M.; Cozzuol, M.; Screen, T. E. O.; Anderson, H. L.; Perry, J. W. Opt. Express 2009, $17,18478$.

(12) Hales, J. M.; Matichak, J.; Barlow, S.; Ohira, S.; Yesudas, K.; Bredas, J. L.; Perry, J. W.; Marder, S. R. Science 2010, 327, 1485.

(13) Barlow, S.; Brédas, J. L.; Getmanenko, Y. A.; Gieseking, R. L.; Hales, J. M.; Kieu, K.; Kim, H.; Marder, S. R.; Norwood, R. A.; Perry, J. W.; Peyghambarian, N.; Risko, C.; Shahin, S.; Zhang, Y. Mater. Horiz. 2014, 1, 577.

(14) Stegeman, G. I.; Stolen, R. H. J. Opt. Soc. Am. B 1989, 6, 652.

(15) Chiao, R.; Garmire, E.; Townes, C. Phys. Rev. Lett. 1964, 13, 479.

(16) Kelley, P. L. Phys. Rev. Lett. 1965, 15, 1005.

(17) Lallemand, P.; Bloembergen, N. Phys. Rev. Lett. 1965, 15, 1010.

(18) Kashyap, R.; Blow, K. J. Electron. Lett. 1988, 24, 47.

(19) Bergano, N. S.; Davidson, C. R. J. Lightwave Techn. 1995, 13, 879.

(20) Essiambre, R. J.; Kramer, G.; Winzer, P. J.; Foschini, G. J.; Goebel, B. J. Lightwave Techn. 2010, 28, 662 .

1886.

(21) Huy, M. C. P.; Baron, A.; Lebrun, S.; Frey, R.; Delaye, P. J. Opt. Soc. Am. B 2010, 27,

(22) Voronin, A. A.; Mitrokhin, V. P.; Ivanov, A. A.; Fedotov, A. B.; Sidorov-Biryukov, D. A.; Beloglazov, V. I.; Alfimov, M. V.; Ludvigsen, H.; Zheltikov, A. M. Laser Phys. Lett. 2010, 7, 46.

(23) Ippen, E. P.; Shank, C. V.; Gustafso.Tk Appl. Phys. Lett. 1974, 24, 190.

(24) Coen, S.; Chau, A. H. L.; Leonhardt, R.; Harvey, J. D.; Knight, J. C.; Wadsworth, W. J.; Russell, P. S. J. J. Opt. Soc. Am. B 2002, 19, 753.

(25) Bethge, J.; Husakou, A.; Mitschke, F.; Noack, F.; Griebner, U.; Steinmeyer, G.; Herrmann, J. Opt. Express 2010, 18, 6230.

(26) Marder, S. R.; Gorman, C. B.; Meyers, F.; Perry, J. W.; Bourhill, G.; Brédas, J. L.; Pierce, B. M. Science 1994, 265, 632.

10703.

(27) Meyers, F.; Marder, S. R.; Pierce, B. M.; Bredas, J. L. J. Am. Chem. Soc. 1994, 116,

(28) Yang, M.; Jiang, Y. Phys. Chem. Chem. Phys. 2001, 3, 4213.

(29) Guo, S.-L.; Li, T.-P.; Wang, T.-B.; Liu, Z.-S.; Cao, T.-D. Opt. Mater. 2007, 29, 494. 25 
(30) Prabhakar, C.; Yesudas, K.; Bhanuprakash, K.; Jayathirtha Rao, V.; Sai Santosh Kumar, R.; Narayana Rao, D. J. Phys. Chem. C 2008, 112, 13272.

(31) Dirk, C. W.; Herndon, W. C.; Cervantes-Lee, F.; Selnau, H.; Martinez, S.; Kalamegham, P.; Tan, A.; Campos, G.; Velez, M.; Zyss, J.; Ledoux, I.; Cheng, L.-T. J. Am. Chem. Soc. 1995, 117, 2214.

(32) Whittall, I. R.; Humphrey, M. G. Organometallics 1995, 14, 5493.

(33) Letard, J.-F.; Montant, S.; Guionneau, P.; Martin, P.; Le Calvez, A.; Freysz, E.; Chasseau, D.; Lapouyade, R.; Kahn, O. Chem. Commun. 1997, 1997, 745.

(34) Diaz-Garcia, M. A.; Ledoux, I.; Duro, J. A.; Torres, T.; Agullo-Lopez, F.; Zyss, J. J. Phys. Chem. 1994, 98, 8761.

(35) DeSalvo, R.; Said, A. A.; Hagan, D. J.; VanStryland, E. W.; SheikBahae, M. IEEE J. Quantum Electron. 1996, 32, 1324.

(36) Tanaka, K. J. Mater. Sci. - Mater. Electron. 2005, 16, 633.

(37) Orr, B. J.; Ward, J. F. Molec. Phys. 1971, 20, 513.

(38) Pierce, B. M. Proc. SPIE 1991, 1560, 148.

(39) Garito, A. F.; Teng, C. C. Proc. SPIE 1986, 0613, 146.

(40) Helfin, J. R.; Garito, A. F. In Electroresponsive Molecular and Polymeric Systems; Skotheim, T. A., Ed.; Marcel Dekker Inc.: New York, 1991, p 1.

(41) Pierce, B. M. In Molecular and Biomolecular Electronics; American Chemical Society: Washington DC, 1994; Vol. 240, p 243.

(42) Dirk, C. W.; Cheng, L.-T.; Kuzyk, M. G. Int. J. Quantum Chem. 1992, 43, 27.

(43) Kuzyk, M.; Dirk, C. Phys. Rev. A 1990, 41, 5098.

(44) Marder, S. R.; Perry, J. W.; Bourhill, G.; Gorman, C. B.; Tiemann, B. G.; Mansour, K. Science 1993, 261, 186.

(45) Wernke, W.; Pfeiffer, M.; Johr, T.; Lau, A.; Grahn, W.; Johannes, H.-H.; Dahne, L. Chem. Phys. 1997, 216, 337.

(46) Mukhopadhyay, S.; Risko, C.; Marder, S. R.; Brédas, J.-L. Chem. Sci. 2012, 3, 3103.

(47) Chai, J.-D.; Head-Gordon, M. Phys. Chem. Chem. Phys. 2008, 10, 6615.

(48) Chai, J.-D.; Head-Gordon, M. J. Chem. Phys. 2008, 128, 084106.

(49) Dunning, T. H. J. Chem. Phys. 1989, 90, 1007.

(50) Gaussian 09, Revision B.01, M. J. Frisch, G. W. Trucks, H. B. Schlegel, G. E. Scuseria, M. A. Robb, J. R. Cheeseman, G. Scalmani, V. Barone, B. Mennucci, G. A. Petersson, H. Nakatsuji, M. Caricato, X. Li, H. P. Hratchian, A. F. Izmaylov, J. Bloino, G. Zheng, J. L. Sonnenberg, M. Hada, M. Ehara, K. Toyota, R. Fukuda, J. Hasegawa, M. Ishida, T. Nakajima, Y. Honda, O. Kitao, H. Nakai, T. Vreven, J. A. Montgomery, Jr., J. E. Peralta, F. Ogliaro, M. Bearpark, J. J. Heyd, E. Brothers, K. N. Kudin, V. N. Staroverov, T. Keith, R. Kobayashi, J. Normand, K. Raghavachari, A. Rendell, J. C. Burant, S. S. Iyengar, J. Tomasi, M. Cossi, N. Rega, J. M. Millam, M. Klene, J. E. Knox, J. B. Cross, V. Bakken, C. Adamo, J. Jaramillo, R. Gomperts, R. E. Stratmann, O. Yazyev, A. J. Austin, R. Cammi, C. Pomelli, J. W. Ochterski, R. L. Martin, K. Morokuma, V. G. Zakrzewski, G. A. Voth, P. Salvador, J. J. Dannenberg, S. Dapprich, A. D. Daniels, O. Farkas, J. B. Foresman, J. V. Ortiz, J. Cioslowski, and D. J. Fox, Gaussian, Inc., Wallingford CT, 2010.

(51) Zojer, E.; Beljonne, D.; Kogej, T.; Vogel, H.; Marder, S. R.; Perry, J. W.; Brédas, J. L. J. Chem. Phys. 2002, 116, 3646.

(52) Ohira, S.; Hales, J. M.; Thorley, K. J.; Anderson, H. L.; Perry, J. W.; Bredas, J. L. J. Am. Chem. Soc. 2009, 131, 6099.

(53) Zojer, E.; Beljonne, D.; Pacher, P.; Bredas, J. L. Chem. Eur. J. 2004, 10, 2668.

(54) Zojer, E.; Wenseleers, W.; Halik, M.; Grasso, C.; Barlow, S.; Perry, J. W.; Marder, S. R.; Brédas, J.-L. ChemPhysChem 2004, 5, 982.

(55) Gieseking, R. L.; Mukhopadhyay, S.; Risko, C.; Brédas, J.-L. ACS Photon. 2014, 1, 261.

(56) Cave, R. J.; Davidson, E. R. J. Phys. Chem. 1988, 92, 614. 
(57) Cave, R. J.; Davidson, E. R. J. Phys. Chem. 1987, 91, 4481.

(58) Ferdinandus, M. R.; Reichert, M.; Ensley, T. R.; Hu, H.; Fishman, D. A.; Webster, S.; Hagan, D. J.; Van Stryland, E. W. Opt. Mater. Express 2012, 2, 1776.

(59) Sheik-Bahae, M.; Said, A. A.; Wei, T.-H.; Hagan, D. J.; Van Stryland, E. W. IEEE J. Quantum Electron. 1990, 26, 760.

(60) In charged systems, the state dipole moments depend on the choice of the origin; however, the differences between state dipole moments do not so long as the same origin is used for both state dipole moments. We select the location of the central atom $\mathrm{X}$ as the origin; since the molecules have S4 symmetry, this is also the center of charge.

(61) The orbital components of the transition dipole moments discussed here include a quantum prefactor of sqrt(2) because the INDO/CI approach uses singlet-adapted states.

(62) Nalwa, H. S. Adv. Mater. 1993, 5, 341.

(63) Tykwinski, R. R.; Gubler, U.; Martin, R. E.; Diederich, F.; Bosshard, C.; Gunter, P. J. Phys. Chem. B 1998, 102, 4451.

(64) Gubler, U.; Bosshard, C. Adv. Polym. Sci. 2002, 158, 123.

(65) Bredas, J. L.; Adant, C.; Tackx, P.; Persoons, A.; Pierce, B. M. Chem. Rev. 1994, 94, 243.

(66) Milam, D. Appl. Opt. 1998, 37, 546.

(67) Hurlbut, W. C.; Lee, Y.-S.; Vodopyanov, K. L.; Kuo, P. S.; Fejer, M. M. Opt. Lett. 2007, 32,668 .

(68) Van Stryland, E. W.; Woodall, M. A.; Vanherzeele, H.; Soileau, M. J. Opt. Lett. 1985, $10,490$.

(69) Because wexc/wge is relatively large in the selenopyrylium polymethines, $\operatorname{Re}(y)$ is enhanced relative to the static (zero-frequency) limit due to pre-resonant enhancement. The computed $\operatorname{Re}(\mathrm{y})$ stat for the $\mathrm{Se}-3 \mathrm{C}$ is approximately 28 times larger than that of $\mathrm{PPh} 4+$, and the larger selenopyrylium polymethines have even larger $\operatorname{Re}(\mathrm{y})$ stat values. 


\section{TOC Graphic}

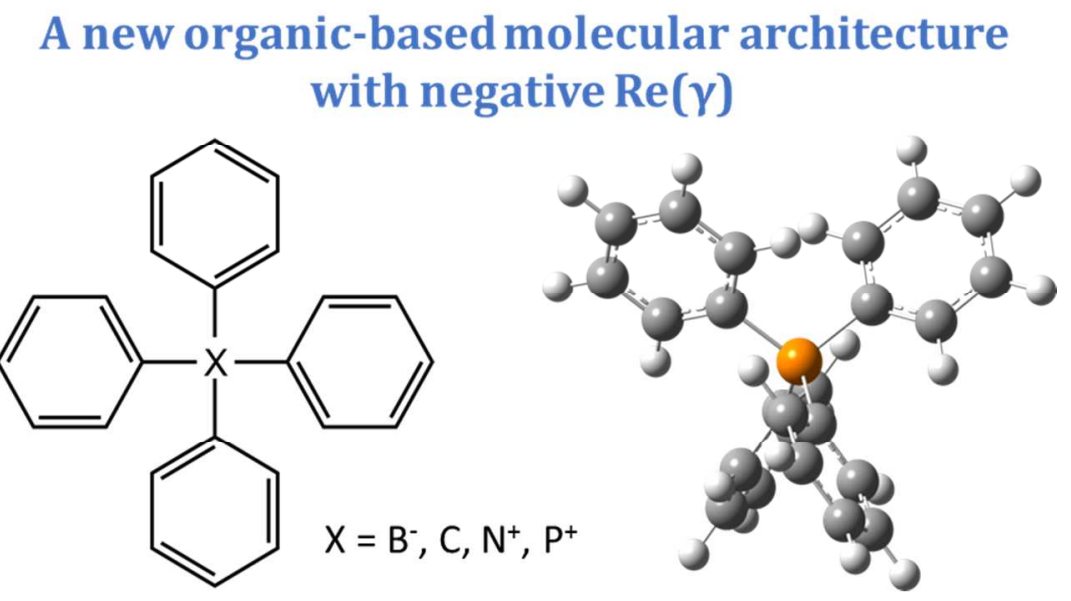

20

21

22

23

24

25

26

27

28

29

30

31

32

33

34

35

36

37

38

39

40

41

42

43

44

45

46

47

48

49

50

51

52

53

54

55

56

57

58

59

60 